Check for updates

Cite this: RSC Adv., 2018, 8, 1647

Received 1st November 2017

Accepted 19th December 2017

DOI: 10.1039/c7ra12032j

rsc.li/rsc-advances

\section{A statherin-derived peptide promotes hydroxyapatite crystallization and in situ remineralization of artificial enamel caries $\dagger$}

\author{
Kun Wang, Di Xiuqing Wang, $\$$ Haoran Li, Sainan Zheng, Qian Ren, Yufei Wang, \\ Yumei Niu, Wei Li, Xuedong Zhou and Linglin Zhang*
}

In situ remineralization of hydroxyapatite on a human tooth enamel surface induced by anti-caries bioactive components is an alternative restorative strategy against dental caries. In this study, a novel biomimetic peptide DE-11, inspired by the salivary phosphoprotein statherin, was developed, and it showed beneficial potentials for the restoration of demineralized tooth enamel in vitro. The peptide DE-11 contained the initial six-peptide sequence of $\mathrm{N}$-terminus of statherin extended by a mineralization hydrophilic tail composed of consecutive acidic amino acids capable of adsorbing calcium and phosphate ions. A strong adsorption capacity of DE-11 to hydroxyapatite was confirmed through Langmuir adsorption isotherm experiment and confocal laser scanning microscopy. Then, the nucleation and crystallization of hydroxyapatite due to DE-11 was characterized by scanning and transmission electron microscopy and selected-area electron diffraction. Moreover, the ability of DE-11 to promote the remineralization of initial enamel caries lesions was further evaluated. Initial lesions were created in bovine enamel blocks, which were then exposed to the peptide solution and finally immersed in artificial saliva. After 7 days, a higher percentage of surface microhardness recovery, a lower mineral loss, a shallower lesion depth, and a higher mineral content were found on the surface of the lesion body in the DE-11 group as compared to that in the negative group using surface microhardness testing, polarized light microscopy, and transverse microradiography; this indicated that DE-11 could induce in situ remineralization of hydroxyapatite on the demineralized enamel surface. Overall, these findings suggest that DE-11 is highly promising as a restorative biomaterial for enamel remineralization in the anti-caries applications.

\section{Introduction}

Unlike other mineralized tissues (i.e., bone, dentine, and cementum) of human body, dental enamel is the hardest acellular tissue and comprises approximately 96\% carbonated hydroxyapatite (HA) mineral with outstanding hardness and flexural strength. ${ }^{\mathbf{1}}$ Enamel is relatively stable in a healthy oral environment, with a dynamic equilibrium between demineralization and remineralization at the tooth-pellicle and plaquesaliva interfaces. ${ }^{2}$ As the outermost layer covering the tooth, enamel is susceptible to be permanently damaged by dental caries, which is a dynamic disease process caused by the

State Key Laboratory of Oral Diseases, National Clinical Research Center for Oral Diseases, Dept. of Cariology and Endodontics, West China Hospital of Stomatology, Sichuan University, Chengdu, Sichuan, China. E-mail: zhll_sc@163.com; Tel: +86-028-85503470

$\dagger$ Electronic supplementary information (ESI) available: Additional experimental data for RP-HPLC, mass spectrum, microscopic appearance of HOK cells, CLSM images, and EDS analysis spectra are included. See DOI: 10.1039/c7ra12032j

† Equal contributors. imbalance between demineralization and remineralization. ${ }^{3}$ It is of particular significance to promote the remineralization of early small caries lesions before they progress into cavities. Recently, various remineralization strategies have been developed using fluoride, electrolytic deposition, hydroxyapatite nanoparticles, amorphous calcium phosphates, and hydrogen peroxide. ${ }^{4-8}$ Moreover, synthetic peptides have been utilized in biomimetic processes for hard tissue remineralization..$^{\mathbf{9 1 1}}$ However, most of these methods failed to be translated to clinical applications due to their adverse reactions, stringent conditions or controversial therapeutic efficacy. ${ }^{\mathbf{1 2}}$ Therefore, the in situ remineralization of HA under physiological oral conditions is a desirable alternative restorative pathway against dental caries.

As enamel is constantly exposed to saliva, this oral fluid plays a crucial role in the caries remineralization process by providing a multitude of proteins important to maintain the integrity of teeth and regulate caries risk. ${ }^{\mathbf{1 3 1 4}}$ Among them, statherin is a small acidic salivary phosphoprotein that functions as an inhibitor of spontaneous precipitation of calcium phosphate salts to maintain a stable supersaturated state of saliva. ${ }^{15}$ In 
addition, statherin is found to be a major contributor to the acquired enamel pellicle, which is a thin proteinaceous film adsorbed on tooth surfaces and slows down enamel demineralization during an acid attack. ${ }^{\mathbf{1 6}}$ Through this mechanism, statherin plays a key role in constituting a reparative and protective environment crucial for the integrity of teeth.

The acidic domain at the N-terminus was previously shown to be important for the binding of statherin to the HA surface. ${ }^{17}$ In vitro studies demonstrated that the $\mathrm{N}$-terminal 15-amino-acid residue of statherin, known as SN15 (DPSpSEEKFLRRIGRFG, where pS denotes a phosphorylated serine) and its analog $\mathrm{SN}_{\mathrm{A}} 15$ (DDDEEKFLRRIGRFG) exhibited high adsorption on the surface of HA. ${ }^{18}$ Furthermore, the N-terminal domain of the protein (residues 1-12) was shown to be in close proximity to the HA surface upon adsorption due to its $\alpha$-helical structure. ${ }^{19}$ More recently, a cysteine-labelled peptide based on the initial six-peptide sequence of N-terminus of statherin has been reported to possess a strong adsorption capacity to the tooth enamel. ${ }^{20}$ In the abovementioned studies, the key amino acid sequences in statherin have been identified that are particularly important for HA adsorption; this reveals the feasibility that peptides based on these key residues may be effective for in situ enamel biomimetic remineralization.

In this study, we aimed at (1) modifying the natural motif sequence derived from statherin to achieve a bifunctional peptide, DE-11, consisting of 11 amino acids capable of tightly adsorbing to HA through the N-terminal 6-amino-acid residue of statherin (DPSPSEEK) and inducing calcified remineralization via extending a tail composed of multiple glutamic acids; (2) exploring the effect of the novel peptide on HA crystallization occurring in vitro; and (3) identifying its potential for in situ remineralization of artificial enamel caries to offer a promising biomimetic agent for anti-caries application.

\section{Materials and experimental methods}

\subsection{Peptide synthesis, purification, and characterization}

The peptide Asp-pSer-pSer-Glu-Glu-Lys-(Glu) $)_{5}$ denoted as DE11 and the initial six-peptide Asp-pSer-pSer-Glu-Glu-Lys, denoted as DK-6, of the N-terminus of statherin were synthesized by GL Biochem (Shanghai, China) using a standard fluorenylmethoxycarbonyl (Fmoc) solid-phase method as previously described. ${ }^{9}$ The synthesized peptides were then purified (>95\%) by reverse-phase high-performance liquid chromatography (RPHPLC; CHTH Sci and Tech, Beijing, China). The peptide sequence and integrity were confirmed by electrospray ionization mass spectrometry (ESI-MS; Shimadzu, Kyoto, Japan).

\subsection{Circular dichroism spectroscopy}

Circular dichroism (CD) spectra were obtained in the range of 190-250 $\mathrm{nm}$ at room temperature using a CD spectrometer (Chirascan, Applied Photophysics, England). The peptide DE-11 solution was diluted with $20 \mathrm{mM}$ HEPES buffer $(\mathrm{pH}=7.4)$ to yield a final concentration of $0.2 \mathrm{mg} \mathrm{ml}^{-1}$. The measurements were conducted after $2 \mathrm{~h}$ and $24 \mathrm{~h}$ of incubation at $37^{\circ} \mathrm{C}$. To investigate the effects of calcium and phosphate ions on the secondary structure of DE-11, the peptide was incubated with $10 \mathrm{mM} \mathrm{CaCl}_{2}$ and $5 \mathrm{mM} \mathrm{Na}_{2} \mathrm{HPO}_{4}$ separately. All spectra were obtained as the average of five scans using a $2 \mathrm{~nm}$ bandwidth and a $1 \mathrm{~nm} \mathrm{~s}^{-1}$ scanning rate. CD spectroscopic data were analyzed using the CDPro software package (JASCO, Tokyo, Japan) and CONTINLL algorithm.

\subsection{Cell proliferation assay}

Effect of DE-11 on cell proliferation was investigated by the Cell Counting Kit-8 (CCK-8, Dojindo, Japan) assay. Human oral keratinocyte (HOK) cells obtained from the American Type Culture Collection (ATCC, 11303) were seeded at $2 \times 10^{3}$ cells per well in 96-well plates and incubated in DMEM (HyClone, Logan, UT, USA) with 10\% fetal bovine serum (Gibco, Grand Island, NY, USA) $(100 \mu \mathrm{l}$ per well) for $24 \mathrm{~h}$. Cells were exposed to DE-11 with the final concentrations of 0, 50, 100, 150, 200, 250, $300,350,400$, and $500 \mu \mathrm{M}$. The cell viability was assessed after $24 \mathrm{~h}$ exposure. Absorbance at $450 \mathrm{~nm}$ was measured using Thermo Scientific Varioskan Flash (Thermo Scientific, Waltham, USA). The absorbance was the average value measured from five wells in parallel for each concentration.

\subsection{Binding capacity of DE-11 to HA}

The peptide DE-11 with concentrations in the range from 50 to $250 \mu \mathrm{M}$ (dissolved in $20 \mathrm{mM}$ HEPES buffer $\mathrm{pH}=7.4$ ) was mixed with $50 \mathrm{mg}$ of HA powder. The mixtures were rotated at $37{ }^{\circ} \mathrm{C}$ overnight and then centrifuged at $14000 \mathrm{rpm}$ for $10 \mathrm{~min}$ to obtain the supernatants. The concentrations of DE-11 in solutions before and after incubation with the HA powder were measured using the Micro BCA protein assay kit (Cwbio, Beijing, China). After calculation, the amount of peptide bound to a unit surface area of HA powder was obtained. After this, the linear adsorption isotherm was plotted using the Langmuir equation (eqn (1)). ${ }^{21,22}$ Eventually, from the resultant best-fit lines, the maximum number of adsorption sites available for DE-11 per gram of $\mathrm{HA}$ and the affinity of DE-11 for HA adsorption sites were calculated.

$$
\frac{C_{\mathrm{eq}}}{Q}=\frac{1}{N K}+\frac{C_{\mathrm{eq}}}{N}
$$

$C_{\text {eq }}=$ equilibrium protein concentration after incubation with HA (mM), $Q=$ amount of protein adsorbed per gram of HA powder $\left(\mu \mathrm{mol} \mathrm{g}{ }^{-1}\right), N=$ the maximum number of adsorption sites per gram of $\mathrm{HA}\left(\mu \mathrm{mol} \mathrm{g}{ }^{-1}\right)$ and $K=$ affinity of the protein molecules for HA adsorption sites $\left(\mathrm{ml} \mu \mathrm{mol}^{-1}\right)$.

\subsection{Binding capacity of FITC-labelled DE-11 to the enamel surface}

Phosphoric acid (37\%) was used to prepare the acid-etched tooth enamel. Bovine permanent incisors without any lesions, cracks or fluoric mottle were polished with sandpapers $(1000$, $1200,2400,3000$, and 4000 grits) to a thickness of approximately $100 \mu \mathrm{m}$. Then, the enamel slices were etched in 37\% phosphoric acid for 45 s. Finally, the samples were washed with sterile 
deionized water to stop the acid etching and sonicated for $5 \mathrm{~min}$.

Fluorescein isothiocyanate (FITC)-labelled DE-11 was synthesized by GL Biochem (Shanghai, China). The FITClabelled DE-11 solution ( $50 \mu \mathrm{M}, 100 \mu \mathrm{l})$ was dropped on both the normal tooth enamel and the acid-etched tooth enamel surfaces. After $30 \mathrm{~min}$, the coated slides were thoroughly washed thrice with sterile deionized water and observed using confocal laser scanning microscope (CLSM, OLYMPUS, Tokyo, Japan) at an excitation wavelength of $488 \mathrm{~nm} .^{23}$

\subsection{In vitro biomineralization and electron microscopy analysis}

The lyophilized peptides DE-11 and DK-6 were dissolved in $20 \mathrm{mM}$ HEPES buffer $(\mathrm{pH}=7.4)$. The mineralization reaction was performed by incubating $\mathrm{CaCl}_{2}$ with $\mathrm{Na}_{2} \mathrm{HPO}_{4}$ in HEPES at $37^{\circ} \mathrm{C}$ for $24 \mathrm{~h}$ in the presence or absence of peptides to achieve the final concentrations of $3.3 \mathrm{mM} \mathrm{CaCl}, 1.6 \mathrm{mM} \mathrm{Na} \mathrm{HPO}_{4}$, and (or) $50 \mu \mathrm{M}$ peptides $(\mathrm{pH}=7.4) .{ }^{24,25}$ After incubation, $10 \mu \mathrm{l}$ of each sample solution (including DE-11, DK-6, and a negative control) was pipetted onto a carbon-coated $\mathrm{Cu}$ TEM grid. The calcium phosphate precipitates were then characterized by scanning electron microscopy (SEM), transmission electron microscopy (TEM) with selected area electron diffraction (SAED), and energy-dispersive X-ray spectroscopy (EDS). SEM images were obtained using a scanning electron microscope (Inspect F, FEI, Eindhoven, The Netherlands) at $20.0 \mathrm{kV}$. In TEM analysis, mineralized suspensions were observed using an FEI Tecnai F20 S-TWIN electron microscope at $120 \mathrm{kV}$.

\subsection{Remineralization effect of DE-11 on artificial enamel caries}

2.7.1. Preparation of tooth enamel samples. Newly extracted bovine permanent incisors free of lesions, cracks or fluoric mottle were cleaned from debris and stored in distilled water with a $0.5 \%$ thymol solution until further use. Crowns were separated from the roots using a diamond-coated band saw with continuous water cooling (Struers Minitom, Struers, Copenhagen, Denmark) to obtain $5 \mathrm{~mm} \times 5 \mathrm{~mm}$ square samples. The enamel surfaces were ground flat and polished sequentially using water-cooled carborundum discs of waterproof silicon carbide paper with various grits $(1000,1500,2000$, 2500, 3000, and 4000 grit; Struers). All polished enamel blocks were then embedded in polymethylmethacrylate and partly painted with two layers of acid-resistant nail varnish leaving an exposed $4 \mathrm{~mm} \times 4 \mathrm{~mm}$ window.

The baseline surface microhardness $\left(\mathrm{SMH}_{0}\right)$ of the prepared enamel blocks was measured at 5 positions using a microhardness tester (Duramin-1/-2, Struers) and a Knoop indenter at a load of $50 \mathrm{~g}$ for $15 \mathrm{~s} .{ }^{9}$ Samples with $\mathrm{SMH}_{0}$ between 320 and 400 Knoop hardness numbers (KHN) were selected for further study.

2.7.2. Carious lesion formation. Initial enamel carious lesions were artificially induced by acid treatment as previously described. ${ }^{10}$ Enamel blocks were submerged in the demineralization solution containing $50 \mathrm{mM}$ acetic acid $(\mathrm{pH}=4.5)$,
$2.2 \mathrm{mM} \mathrm{KH}_{2} \mathrm{PO}_{4}, 2.2 \mathrm{mM} \mathrm{Ca}\left(\mathrm{NO}_{3}\right)_{2}, 5.0 \mathrm{mM} \mathrm{NaN}_{3}$, and $0.5 \mathrm{ppm}$ $\mathrm{NaF}$ at $37^{\circ} \mathrm{C}$ for 3 days under continuous, low-speed magnetic stirring $(100 \mathrm{rpm})$. Post-demineralization surface microhardness $\left(\mathrm{SMH}_{1}\right)$ was measured as described above. A total of 40 enamel blocks with $\mathrm{SMH}_{1}$ between 130 and $220 \mathrm{KHN}$ were then selected for subsequent remineralization assay. The exposed windows on these samples were sealed with a film and two layers of acid-resistant nail varnish; this left an exposed area of $4 \mathrm{~mm} \times 2 \mathrm{~mm}$.

2.7.3. Remineralization of tooth enamel in artificial saliva. All 40 enamel samples after demineralization were randomly divided into four groups (10 specimens per group) and treated with the following agents: the positive control group: $1000 \mathrm{ppm}$ $\mathrm{NaF}$; the peptide DE-11 group: $50 \mu \mathrm{M} \mathrm{DE}-11$; the peptide DK-6 group: $50 \mu \mathrm{M}$ DK-6; and the negative control group: $20 \mathrm{mM}$ HEPES. Each specimen was exposed to the abovementioned solutions for $30 \mathrm{~min}$ at room temperature. Then, they were rinsed three times with deionized water, followed by immersion in artificial saliva at $37{ }^{\circ} \mathrm{C}$ for 3 days and 7 days. The artificial

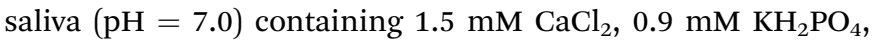
$130 \mathrm{mM} \mathrm{KCl}, 1 \mathrm{mM} \mathrm{NaN}_{3}$, and $20 \mathrm{mM}$ HEPES was freshly prepared daily. ${ }^{\mathbf{2 6}}$

2.7.4. Surface microhardness analysis. SMH of each sample was measured again after remineralization $\left(\mathrm{SMH}_{2}\right)$ for 3 days and 7 days using 5 new indentations located at least 100 $\mu \mathrm{m}$ away from those used to measure $\mathrm{SMH}_{0}$ and $\mathrm{SMH}_{1}$. The percentage surface microhardness recovery (SMHR\%) of four groups was then calculated as $\mathrm{SMHR} \%=\left(\mathrm{SMH}_{2}-\mathrm{SMH}_{1}\right) /$ $\left(\mathrm{SMH}_{1}-\mathrm{SMH}_{0}\right) \times 100 \% .^{27}$

2.7.5. Polarized light microscopy (PLM) and transverse microradiography (TMR) analysis. After 7 days of remineralization treatment, the windows on enamel surfaces were cut into $300 \mu \mathrm{m}$ thick slices using a diamond-coated band saw (Struers). All the sections were polished to a thickness of about $100 \mu \mathrm{m}$, verified using a digital micrometer (Mitu-toyo, Tokyo, Japan). Each slice was then cleaned by 5 min sonication (Jiahui, Suzhou, China) to remove any dust particles. Slices immersed in distilled deionized water were examined using a polarized light microscope (ECLIPSE ME600L, Nikon, Tokyo, Japan). Next, the slices were fixed on Plexiglas sides in a transverse microradiography (TMR) sample holder (Inspekot Research Systems BV, Amsterdam, The Netherlands) and microradiographed alongside an aluminum calibration step wedge with 14 steps using a monochromatic CuK X-ray source (Philips, Eindhoven, The Netherlands) operated at $20 \mathrm{kV}$ and $20 \mathrm{~mA}$ for a $25 \mathrm{~s}$ exposure time. Mineral loss, lesion depth, and mineral content in different enamel layers were assessed using the imaging software (Transversal Microradiography Software 2006, Inspektor Research Systems BV, Amsterdam, The Netherlands). Herein, ten TMR traces along each slice were measured, including five traces within the areas that were not exposed to artificial saliva and five within the exposed areas.

\subsection{Statistical analysis}

Statistical analyses were performed by the SPSS 19.0 software (Chicago, IL, USA) and GraphPad Prism software (San Diego, 
CA, USA). ANOVA was used to analyze the inter-group differences, and the Student-Newman-Keuls test was applied to compare the individual groups. Students paired $t$ test was used to compare the mineral loss, lesion depth, and mineral content of the surface layer and lesion body among all the groups before and after the remineralization treatment. $P$ value of less than 0.05 was considered statistically significant.

\section{Results}

\subsection{DE-11 design and characterization}

The peptide DE-11 was designed to feature a HA binding motif and a stretch of five consecutive negatively charged glutamic acids, which was expected to induce adsorption of calcium and thus promote mineralization. The initial N-terminal 6-aminoacid residue of statherin was selected as the HA anchoring motif, denoted as DK-6, which was also used as a control to assess the remineralization effect of DE-11. To confirm the structure of synthesized peptides, RP-HPLC analysis was performed. It was found that the characteristic peaks of peptides DE-11 and DK-6 were at $10.88 \mathrm{~min}$ and $13.96 \mathrm{~min}$, respectively, and their purities were more than 95\% (Fig. S1 $\dagger$ ). Moreover, the mass spectra showed a high peak around the theoretical molecular weights of DE-11 and DK-6 (Fig. S1†). These results confirmed the successful synthesis of both peptides.

CD measurement was performed to determine the secondary structure of DE-11. The result showed that DE-11 had a higher percentage of $\beta$-sheet, accounting for $36 \%$. A total of $58.1 \% \beta$ sheet and $\beta$-turn was confirmed in the structure of DE- 11 . Moreover, the CD spectrum with no obvious change after $24 \mathrm{~h}$ confirmed the desirable structural stability of DE-11 (Fig. 1A). Furthermore, CD spectra were obtained in the presence of $\mathrm{Ca}^{2+}$ and $\mathrm{HPO}_{4}{ }^{2-}$, which significantly altered the structure of the peptide DE-11 (Fig. 1B). This result indicated that DE-11 in the solution could interact with calcium and phosphate ions.

\subsection{Effect of DE-11 on cell viability}

In this study, HOK cells were treated with various concentrations of DE-11 in the range from 50 to $500 \mu \mathrm{M}$. After a $24 \mathrm{~h}$ treatment, the effect of DE-11 on the cell viability was examined by the CCK-8 assay. As shown in Fig. 1C, there was no significant difference between the DE-11-treated groups and non-treated control groups $(P>0.05)$. Moreover, the microscopic appearance of DE-11-treated cells indicated no changes in size and shape, and the cells closely resembled normal control cells; this indicated that DE-11 with low cytotoxicity had the potential for further study (Fig. S2 $\dagger$ ).

\subsection{Adsorption capability of DE-11 on HA and demineralized tooth enamel}

The Langmuir adsorption theory was employed to obtain the binding affinity of DE-11 to HA. DE-11 solutions with different concentrations were prepared, and a certain amount of HA powder was added to them before centrifugation. Then, the amount of DE-11 adsorbed on HA was calculated by the decrease of DE-11 in the solution. The adsorption isotherms of DE-11 fit the Langmuir model well $\left(R^{2}\right.$ value $>0.98$; Fig. 2$)$. The binding affinity of DE-11 for HA adsorption sites was $3.77 \times 10^{4}$ $\mathrm{M}^{-1}$, and the maximum number of adsorption sites per gram of HA available for binding to DE-11 was $1.77 \times 10^{-2} \mathrm{~mol} \mathrm{~g}^{-1}$.

To further evaluate the adsorption behavior of DE-11 on tooth enamel, we dropped FITC-labelled DE-11 onto the intact enamel surfaces of bovine permanent incisors and

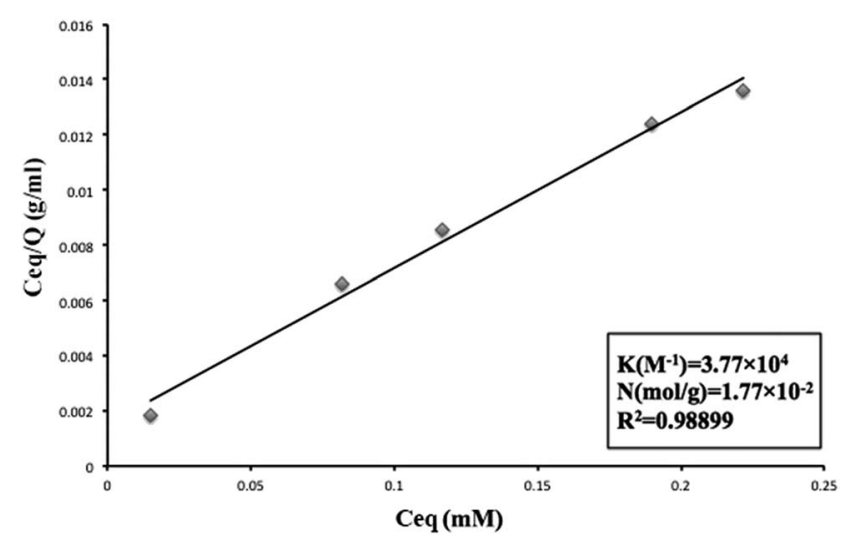

Fig. 2 Linear adsorption isotherms of DE-11. Protein-HA adsorption data fit the Langmuir model well. The affinity of DE-11 molecules for $\mathrm{HA}$ adsorption sites $(K)$ and the maximum number of adsorption sites per gram of HA $(N)$ were calculated. $R^{2}$ is the correlation coefficient obtained for linear adsorption isotherms.

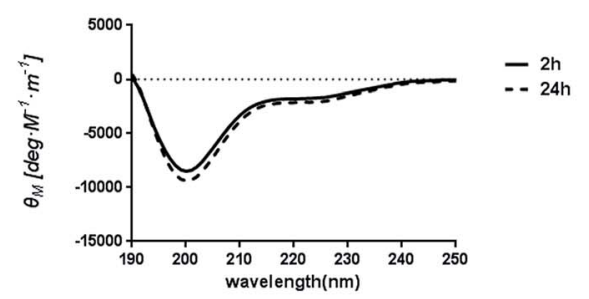

A

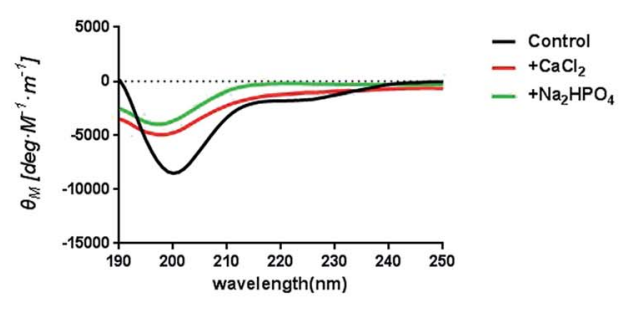

B

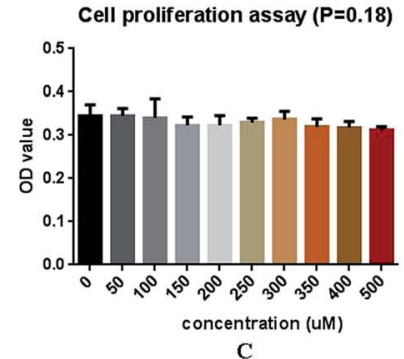

Fig. 1 Circular dichroism and cell viability analysis of DE-11. Peptide DE-11 was dissolved in $20 \mathrm{mM} \mathrm{HEPES} \mathrm{(pH=7.4).} \mathrm{CD} \mathrm{spectra} \mathrm{of} \mathrm{DE-11} \mathrm{after}$ $2 \mathrm{~h}$ (full line) and $24 \mathrm{~h}$ (broken line) of incubation (A). $\mathrm{CD}$ spectra of $\mathrm{DE}-11$ after the addition of $\mathrm{CaCl}_{2}$ (red line) and $\mathrm{Na}_{2} \mathrm{HPO}_{4}(\mathrm{green}$ line), in comparison with those of DE-11 without $\mathrm{Ca}^{2+}$ and $\mathrm{HPO}_{4}{ }^{2-}$ (black line) (B). The effect of HOK cell viability of DE-11 at various concentrations determined by the CCK- 8 assay. Data are presented as the mean \pm SD (C). 
demineralized enamel samples. The samples were then thoroughly rinsed with sterile deionized water, dried at room temperature, and then observed by CLSM. As shown in Fig. S3A, $\dagger$ no fluorescence was found in the control sample. On the contrary, green fluorescence could be observed after the treatment of FITC-labelled DE-11 (Fig. S3B $\dagger$ ). Moreover, the demineralized enamel sample treated by FITC-labelled DE-11 was fully covered with fluorescence, which was much stronger than that in the normal enamel sample treated by FITC-labelled DE-11 (Fig. S3C $\dagger$ ).

\subsection{DE-11 promotes HA mineralization in vitro}

HA crystallization is a crucial process in remineralization. To assess the effect of DE-11 on calcium phosphate mineralization, an additional control peptide, DK-6, lacking the stretch of the Glu residues was synthesized and expected to elucidate the contribution of the Glu tail on HA mineralization. Electron microscopy was used to compare the effects of the synthesized peptides on the nucleation and crystallization of HA after $24 \mathrm{~h}$ of incubation at $37^{\circ} \mathrm{C}$. At first, SEM was applied to observe the morphology of the particles formed in the presence of DE-11, DK-6, and control samples after $24 \mathrm{~h}$ of incubation. Spherical particles were observed in both the control and DK-6 groups with little differences in shape and size (Fig. 3A and C). For the DE-11 group, however, the sample showed a completely different morphology, characterized as significantly small crystals with rough, spicular surfaces (Fig. 3B). After this, TEM showed high magnification images of all the samples, demonstrating that thin plate-like particles formed in the control group (Fig. 4A). However, the presence of DE-11 resulted in obvious changes in the morphology of the HA crystals, shifting to needle-shaped or prismatic morphology (Fig. 4C). Compared to the case of the DK-6 sample, the crystals were found to be much longer and thicker in the DE-11 sample (Fig. 4C and E). Furthermore, these findings were confirmed by SAED. SAED analysis of the DK-6 and negative control samples was consistent with randomly oriented HA crystals, whereas in the presence of DE-11, SAED showed narrow arcs corresponding to 002 and 004 reflections, indicating that the crystals of HA were aligned parallel to the $c$-axis direction (Fig. 4D). Ultimately, EDS

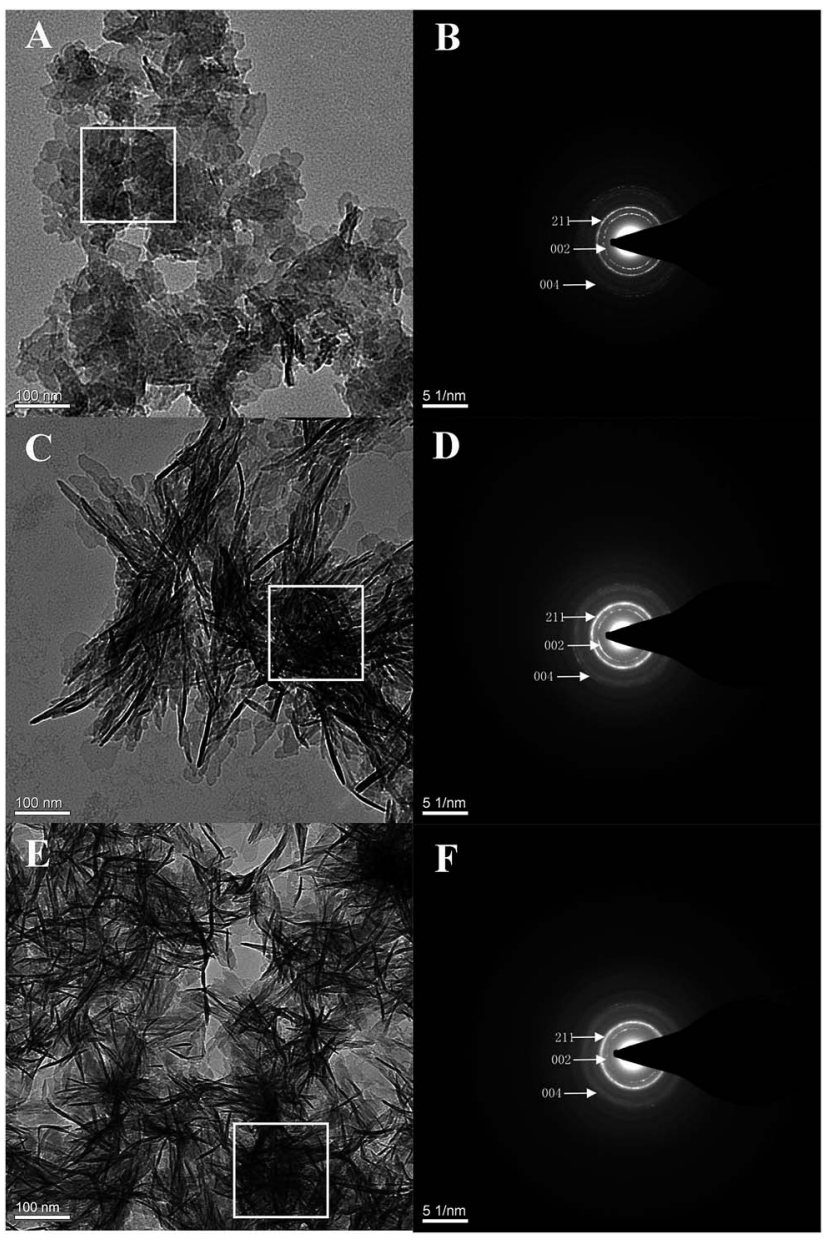

Fig. 4 TEM images of calcium phosphate minerals and related SAED patterns after $24 \mathrm{~h}$ of incubation. Calcium phosphate control group: morphology (A) and SAED pattern (B). DE-11 group: morphology (C) and SAED pattern (D). DK-6 group: morphology (E) and SAED pattern (F). The mineral structure consisting of needle-like crystals was clearly observed in the presence of DE-11.

analysis showed that crystals formed in the DE-11 sample had a $\mathrm{Ca} / \mathrm{P}$ ratio of 1.64 , which approximated the theoretical $\mathrm{Ca} / \mathrm{P}$ ratio for $\mathrm{HA}$, whereas ratios of 1.51 and 1.41 were obtained from the DK-6 and control samples, respectively (Fig. S4†).

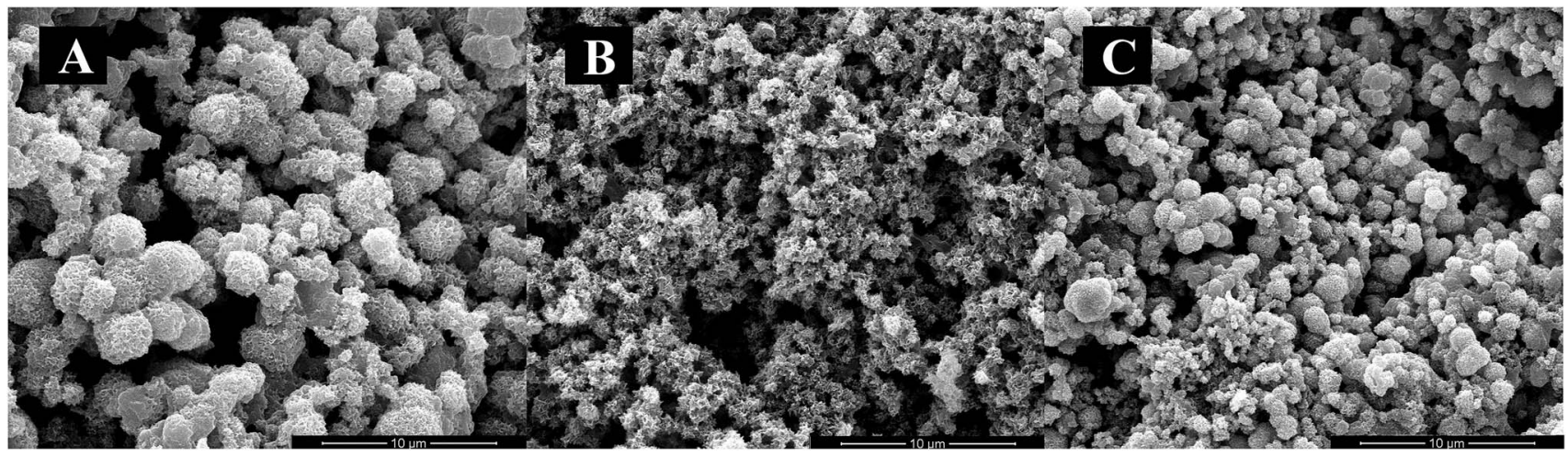

Fig. 3 SEM images of calcium phosphate minerals in the control group (A), DE-11 group (B), and DK-6 group (C) after $24 \mathrm{~h}$ of incubation. Spherical particles were formed in both the DK- 6 and the calcium phosphate control samples, whereas a different morphology characterized as smaller crystals with rough and spicular surfaces was observed in the DE-11 sample. 


\subsection{DE-11 promotes remineralization of initial enamel caries in vitro}

The demineralized enamel blocks treated with and without peptides were immersed in artificial saliva for different time periods ( 3 days and 7 days). To evaluate the mechanical properties of the remineralized enamel samples after treatment, the SMH was detected using the Knoop hardness test. After demineralization, the SMH of all the samples decreased by about $45 \%$ and showed no significant differences among different groups (Fig. 5A). After 7 days of remineralization, there was no significant difference in $\mathrm{SMH}$ between the negative control group and the demineralized enamel samples. On the other hand, after 3 days of remineralization, the SMH recovery (SMHR\%) of samples in the DE-11 and NaF groups was significantly higher than that in the HEPES group $(P<0.05$; Fig. 5B). More importantly, SMHR\% increased remarkably in the DE-11 group after 7 days of treatment (Fig. 5C). However, SMHR\% showed no significant difference between the DK-6 and HEPES groups after remineralization $(P>0.05)$.

PLM demonstrated the expected initial caries lesions induced by controlled demineralization before remineralization treatment, in which the enamel surface layer showed positive birefringence because of the relatively low mineral content. After 7 days of remineralization, a surface layer with a relatively high mineral content, exhibiting negative birefringence, was obviously visible on the enamel samples treated with DE-11 and $\mathrm{NaF}$, but the remineralization zone was thinner on DK-6-treated enamel samples (Fig. 6A-C). In contrast, no significant change was observed in the samples treated with HEPES before and after treatment; this indicated that no remineralization occurred (Fig. 6D). Moreover, the lesions were shown to become significantly shallower after remineralization in the presence of DE-11 and NaF than those in the DK-6 and HEPES groups (Fig. 6). These findings provided the evidence that DE-11 and NaF promoted the remineralization of artificial enamel caries.

Furthermore, the abovementioned results were supported by TMR measurements of mineral loss and lesion depth before and after remineralization. Consistent with the PLM results, the mineral loss and lesion depth after treatment were highest in the HEPES group, intermediate in the samples treated with DK6, and lowest in the DE-11 and NaF groups (Fig. 7A and B). Fig. 7C further exhibits more detailed information about the mineral content at different depths after remineralization for all groups. All samples in different groups showed similar mineral composition in the region extending from the enamel surface to a depth of $20 \mu \mathrm{m}$. Samples treated with NaF and DE-11 showed significantly more mineral content than those in the HEPES groups from the depths of $30 \mu \mathrm{m}$ to $125 \mu \mathrm{m}$, corresponding to the lesion. However, at all the depths examined, the DE-11 group showed a greater mineral content than the DK-6 samples. The mineral content in all the groups was similar at depths beyond $130 \mu \mathrm{m}$.

\section{Discussion}

In an attempt to prevent the progression of caries and improve dental aesthetics, strength, and function, recent studies have been focused on managing non-cavitated caries lesions noninvasively through biomimetic approaches. Inspired by the function of enamel matrix proteins (EMPs) in tooth formation, various peptides, including amelogenin and its functional domains, such as leucine-rich amelogenin peptide, were reported to play essential roles in enamel biomimetic formation.,28,29 However, their capacity to stabilize bioavailable calcium and phosphate ions from saliva and their effect on remineralizing the enamel subsurface lesions need to be further validated in situ and then ultimately in randomized, controlled clinical trials. Therefore, the means for improving the biomimetic peptides used to stabilize, deliver, and control remineralization should be investigated.

Biomineralization proceeds through interactions of mainly calcium and phosphate ions with extracellular matrix molecules that are facilitated by anionic proteins rich in aspartic or glutamic acids. ${ }^{30,31}$ The capability of controlling the formation of inorganic crystals using peptides would be a valuable tool for the restoration of enamel. In addition, enhancement of the HAbinding property of peptides is an essential factor for promoting in situ enamel biomimetic remineralization. Herein, we studied and designed a novel peptide DE-11 inspired by statherin to strongly bind calcium and phosphate ions and induce remineralization on the demineralized enamel surface.

To quantify the adsorption capability of DE-11 on HA, we initially performed Langmuir adsorption isotherm experiment and calculated the binding affinity of DE-11 over HA to be

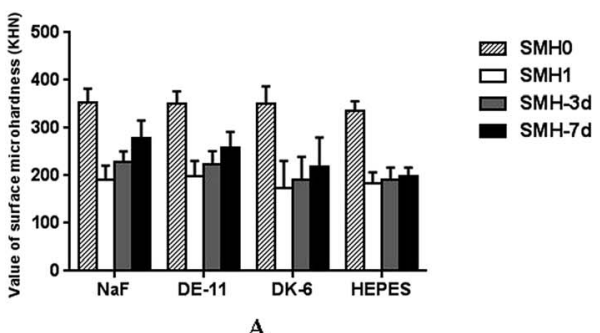

A

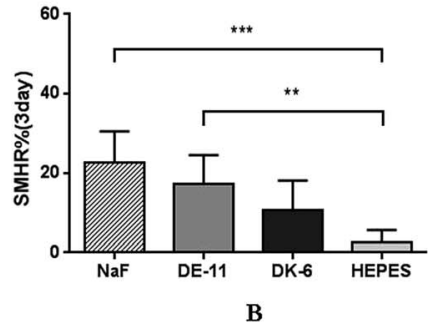

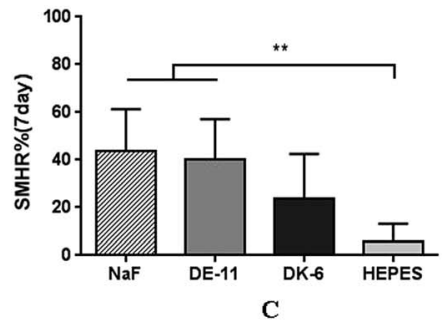

Fig. $5 \mathrm{SMH}$ analysis of the enamel samples after the remineralization assay. The SMH of the NaF group, DE-11-treated group, DK-6-treated group, and HEPES group after immersion in artificial saliva for 3 and 7 days as compared to that of the original enamel samples (A). SMHR\% of the NaF group, DE-11-treated group, DK-6-treated group, and HEPES group after being immersed in artificial saliva for 3 days (B) and for 7 days (C). Data are presented as the mean \pm SD. The statistical analysis was performed using ANOVA followed by the Student-Newman-Keuls test $(* * P<$ $0.01, * * * P<0.001)$. 


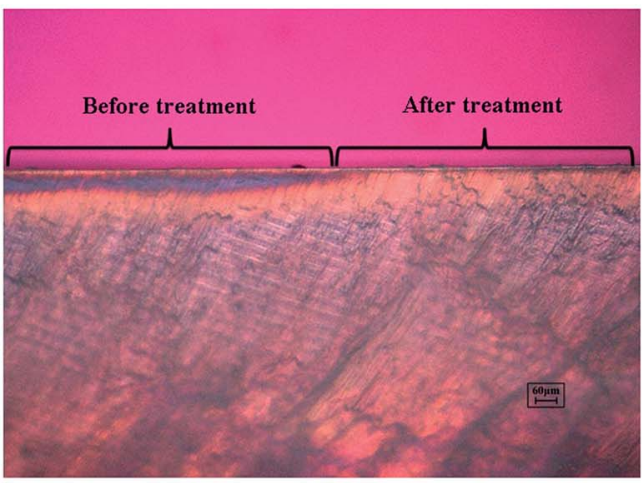

A

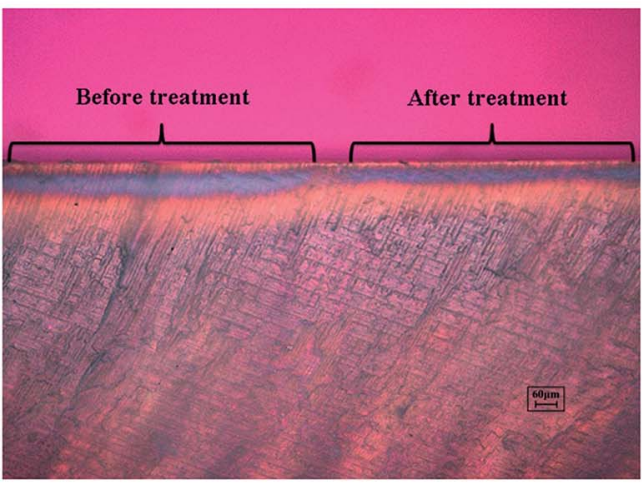

C

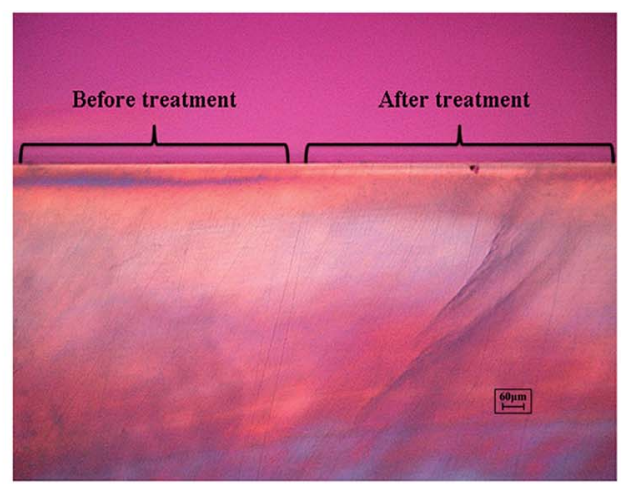

B

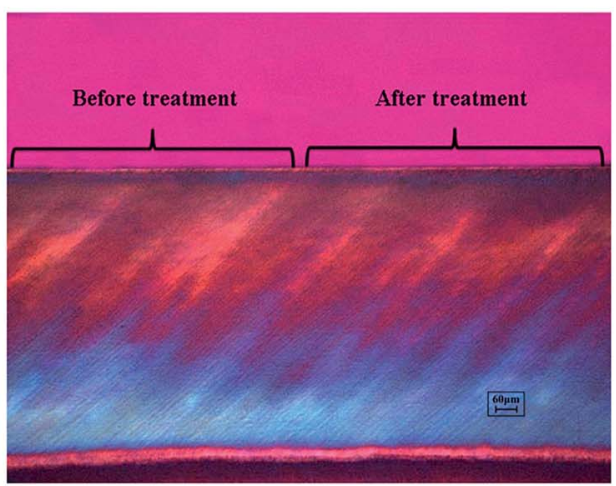

D

Fig. 6 PLM images of the enamel sections before and after remineralization treatment in the presence of $\mathrm{NaF}(\mathrm{A}), \mathrm{DE}-11$ (B), DK-6 (C) or HEPES alone (D).

$3.77 \times 10^{4} \mathrm{M}^{-1}$, which was notably higher than that of statherin, as previously reported. ${ }^{22}$ In addition, the $N$ value for DE-11 was much higher than that for statherin reported in a previous study, ${ }^{22}$ which suggested a preferential HA binding motif on DE11 as compared to that on statherin. Furthermore, FITClabelled DE-11 was dropped on both the normal enamel and demineralized enamel surfaces to verify its binding capability through CLSM analysis. As expected, the fluorescence dispersion on the demineralized enamel sample was strong and uniform, indicating that the adsorption amount of DE-11 on the normal enamel surface was lower than that on the demineralized samples. This result suggested that DE-11 could easily be adsorbed on the enamel surface, especially on the demineralized enamel; this provided a potential possibility of in situ remineralization for anti-caries application.

The specific structure of peptides plays important roles in the formation of HA. ${ }^{32}$ Based on CD measurements, the secondary structure of DE-11 was mainly composed of $\beta$-sheet and $\beta$-turn, which were believed to form biomimetic scaffolds in mineral formation with crystalline orientation. ${ }^{33}$ For the polar

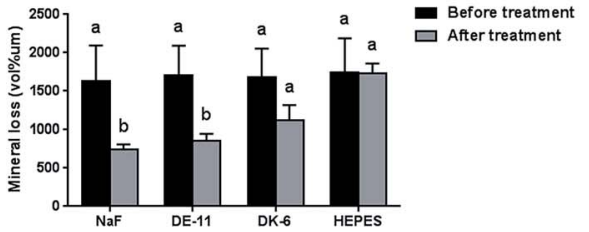

A

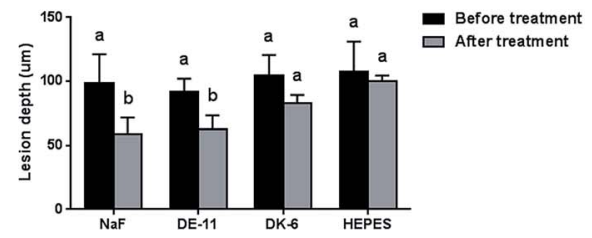

B

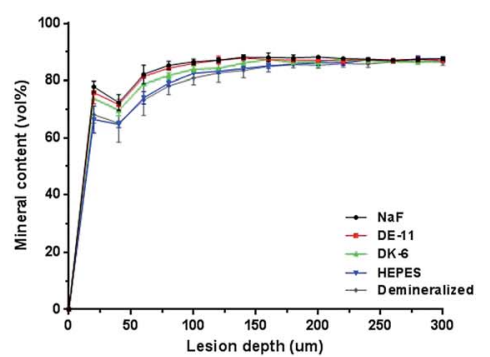

C

Fig. 7 TMR analysis of enamel sections before and after remineralization treatment in the presence of NaF, DE-11, DK-6 or HEPES alone. Mineral loss of enamel blocks (A), lesion depth of enamel blocks (B), and mineral content (vol\% $\mu \mathrm{m})$ vs. depth ( $\mu \mathrm{m})$ for lesions (C). Data are presented as the mean $\pm S D$. The statistical analysis was performed using the Students paired $t$ test. Bars labelled with different letters are significant differences $(P<0.05)$. 
amino acids, consecutive glutamic acids composing the tail of DE-11 contain carboxyl groups in the side chain. The ordered and concentrated carboxyl groups due to $\beta$-sheet formation may be important for hydroxyapatite nucleation. The effect of calcium and phosphate ions on the conformational state of DE11 , as measured by $\mathrm{CD}$, suggested that the interaction of DE-11 with calcium and phosphate ions might create a local supersaturation of ions that would consequently promote HA crystallization. ${ }^{34}$ To verify this hypothesis, we thereby explored whether DE-11 would have an effect on HA mineralization in vitro. For this part, to further validate the in vitro system, we compared the mineralizing effect of DK-6, the natural motif sequence consisting of N-terminal 6-amino-acid residue of statherin. Notably, our TEM results demonstrated that the presence of DE-11 in the reaction resulted in bundles of HA crystals, which were much longer and thicker than that in DK-6, providing direct evidence of the crystalline HA formation enhanced by DE-11. Considering that the thin plate-like particles with random orientation were observed in the negative control sample without a peptide, DE-11 might not mediate the transformation of amorphous calcium phosphate directly, but guide the formation of bundles of well-aligned needle-like apatitic crystals and promote the orientation of HA crystallization. Moreover, the abovementioned results suggest that the tight adsorption of DE-11 to enamel makes it a perfect template for the crystallization of calcium and phosphate ions, and then the new HA crystals are arranged in a well-organized order subsequently.

Next, we explored the potential of DE-11 for the remineralization of initial caries lesions in vitro. In this study, bovine enamel was used as a substitute for human enamel because of the similar chemical composition and physical features between two types of enamels. ${ }^{35}$ In addition, bovine enamel is easier to obtain, and it provides a larger surface area with a more uniform enamel thickness than the human enamel. ${ }^{36}$ As the most widely used remineralizing agent in the treatment of enamel caries, fluoride was chosen to be the positive control to compare the remineralization effect with the peptides. To ensure comparable acid-tolerance of enamel surfaces, all samples for remineralization assay were selected following the inclusion criteria of SMH before and post demineralization. After the remineralization treatment, the $\mathrm{SMH}$ showed no significant difference between the negative control group and the demineralized enamel samples; this indicated that the use of artificial saliva alone could not improve the hardness of the demineralized enamel. SMH analysis demonstrated that the SMHR\% of enamel blocks treated with DE-11 was significantly higher than that of the samples treated with HEPES. Although the DE-11 samples showed a little lower SMHR\% as compared to the NaF group after 3 days of treatment, the difference between them was not significant and decreased obviously after 7 days. As the golden standard technique, TMR was then performed to quantitate the remineralization effect of enamel after treatment with NaF, DE-11, DK-6, and HEPES alone. ${ }^{37}$ TMR analysis showed that mineral loss, lesion depth, and mineral content of the surface layer and lesion body after 7 days of remineralization were similar in the samples treated with DE-11 and NaF. Moreover, the samples in the DE-11 and NaF groups presented a higher mineral content than the samples in the DK6 and HEPES groups at the depths of $30 \mu \mathrm{m}$ to $125 \mu \mathrm{m}$. PLM results were consistent with the TMR results; this suggested that DE-11 possessed a desirable remineralization effect on initial enamel caries lesions in vitro. When compared with NaF, although fluoride is known to reduce the incidence of caries, chronic excessive fluoride exposure can adversely affect tooth growth through interactions with the developing ameloblasts or interrupting the nucleation of apatite crystals, even resulting in fluorosis. ${ }^{38,39}$ Therefore, the peptide DE-11 could be developed as a promising substitute for fluoride-based strategies.

Based on the present results, we demonstrated the feasibility to develop a promising therapeutic peptide derived from bioactive salivary protein for enamel remineralization. Some bioactive salivary proteins, including lysozyme, lactoferrin, and lactoperoxidase, alone or in combinations have been incorporated into oral health care products such as dentifrices, mouthrinses, moisturizing gels, and chewing gums. ${ }^{\mathbf{4 0 , 4 1}}$ Therefore, it seems sensible and significant to further develop salivary biomimetic peptides for anti-caries clinical applications. In our future study, lots of work will be carried out to explore the functional mechanism and efficient conditions of DE-11 through more closely simulating natural processes in the oral cavity.

\section{Conclusions}

In this study, we demonstrated that a novel peptide with low cytotoxicity derived from salivary statherin, DE-11, was capable of adsorbing strongly to the enamel surface while leaving an anionic functional tail free to induce calcium phosphate mineralization. Furthermore, we identified the potential of DE11 for promoting the HA crystallization and in situ remineralization of artificial enamel caries in vitro (Fig. 8). Our results provide the information for further in vivo studies to optimize and adapt this peptide for the biomimetic management of early caries.

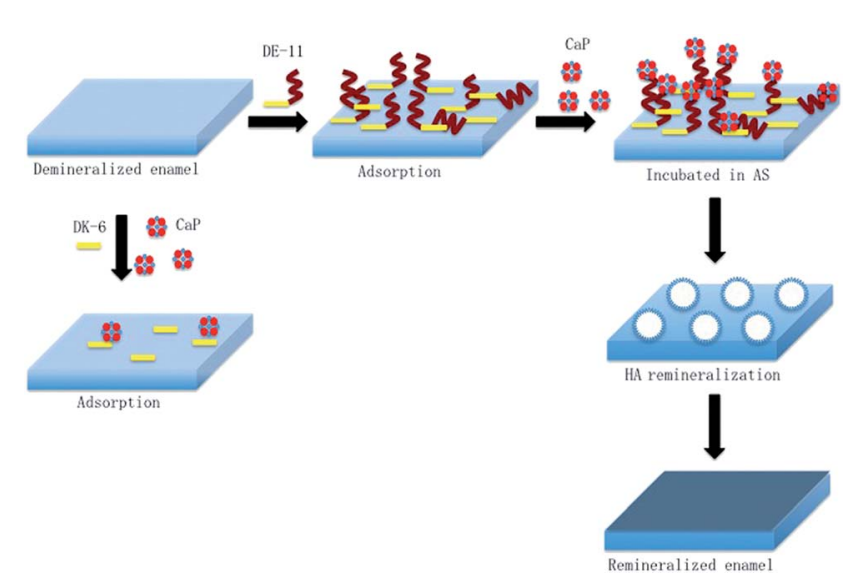

Fig. 8 Schematic of the adsorption of DE-11 on the demineralized enamel surface for remineralization restoration. 


\section{Conflicts of interest}

All authors declare no conflict of interest.

\section{Acknowledgements}

This work was supported by the National Natural Science Foundation of China under grants 81771062 and 81470734 .

\section{Notes and references}

1 H. F. Chen, Z. Y. Tang, J. Liu, K. Sun, S. R. Chang, M. C. Peters, J. F. Mansfield, A. Czajka-Jakubowska and B. H. Clarkson, Adv. Mater., 2010, 18, 1846.

2 T. Aoba, Oral. Dis., 2004, 10, 249.

3 J. D. Featherstone, Aust. Dent. J., 2008, 53, 286.

4 Y. Fan, Z. Sun and J. Moradian-Oldak, Caries Res., 2009, 43, 132.

5 W. Ye and X. X. Wang, Mater. Lett., 2007, 61, 4062.

6 Y. R. Cai and R. K. Tang, J. Mater. Chem., 2008, 18, 3775.

7 M. S. Tung and F. C. Eichmiller, Comp. Cont. Educ. Dent., 2004, 25, 9.

8 K. Yamagishi, K. Onuma, T. Suzuki, F. Okada, J. Tagami, M. Otsuki and P. Senawangse, Nature, 2005, 433, 819.

9 X. P. Lv, Y. Yang, S. L. Han, D. X. Li, H. X. Tu, W. Li, X. D. Zhou and L. L. Zhang, Arch. Oral Biol., 2015, 60, 1482. 10 Y. Yang, X. P. Lv, W. Shi, J. Y. Li, D. X. Li, X. D. Zhou and L. L. Zhang, J. Dent. Res., 2014, 93, 520.

11 Y. Cao, W. Liu, T. Ning, M. L. Mei, Q. L. Li, E. C. Lo and C. H. A. Chu, Clin. Oral. Investig., 2014, 18, 873.

12 M. Tellez, J. Gomez, S. Kaur, I. A. Pretty, R. Ellwood and A. I. Ismail, Community. Dent. Oral. Epidemiol., 2013, 41, 79.

13 M. S. Lamkin and F. G. Oppenheim, Crit. Rev. Oral Biol. Med., 1993, 4, 251.

14 C. J. Whittaker, C. M. Klier and P. E. Kolenbrander, Annu. Rev. Microbiol., 1996, 50, 513.

15 M. W. Dodds, D. A. Johnson and C. K. Yeh, J. Dent., 2005, 33, 223. 16 X. Gao, S. Jiang, D. Koh and C. Y. Hsu, Periodontology 2000, 2016, 70, 128.

17 A. Shimotoyodome, H. Kobayashi, I. Tokimitsu, T. Matsukubo and Y. Takaesu, Caries Res., 2006, 40, 403.

18 M. H. Misbah, M. Espanol, L. Quintanilla, M. P. Ginebra and J. C. Rodríguezcabello, RSC Adv., 2016, 6, 31225.

19 R. Goobes, G. Goobes, W. J. Shaw, G. P. Drobny, C. T. Campbell and P. S. Stayton, Biochemistry, 2007, 46, 4725.
20 Y. X. Yang, B. Yang, M. Z. Li, Y. N. Wang, X. Yang and J. S. Li, Biomed. Mater., 2017, 12, 025007.

21 M. Kresak, E. C. Moreno, R. T. Zahradnik and D. I. Hay, J. Colloid Interface Sci., 1977, 59, 283.

22 D. I. Hay and E. C. Moreno, J. Dent. Res., 1979, 58, 930.

23 Z. B. Huang, X. Shi, J. Mao and S. Q. Gong, Sci. Rep., 2016, 6, 38410.

24 A. S. Deshpande and E. Beniash, Cryst. Growth Des., 2008, 8, 3084 .

25 S. Hosseini, H. Naderi-Manesh, D. Mountassif, M. Cerruti, H. Vali and S. Faghihi, J. Biol. Chem., 2013, 288, 7885.

26 J. M. TenCate and P. P. E. Duijsters, Caries Res., 1982, 16, 201.

27 J. A. Cury, G. S. Simões, A. A. Del Bel Cury, N. C. Goncalves and C. P. M. Tabchoury, Caries Res., 2005, 39, 255.

28 E. Le Norcy, S. Y. Kwak, F. B. Wiedemann-Bidlack, E. Beniash, Y. Yamakoshi, J. P. Simmer and H. C. Margolis, J. Dent. Res., 2011, 90, 1091.

29 F. Shafiei, B. G. Hossein, M. M. Farajollahi, M. Fathollah, B. Marjan and J. K. Tahereh, Scanning, 2015, 37, 179.

30 A. L. Boskey, J. Cell. Biochem., 1998, 30-31, 83.

31 L. Addadi and S. Weiner, Angew. Chem., Int. Ed., 1992, 31, 153.

32 A. Takeuchi, C. Ohtsuki, M. Kamitakahara, S. I. Ogata and T. Miyazaki, J. Mater. Sci.: Mater. Med., 2008, 19, 387.

33 J. Kirkham, A. Firth, D. Vernals, N. Boden, C. Robinson, R. C. Shore, S. J. Brookes and A. Aggeli, J. Dent. Res., 2007, 86, 426.

34 E. Beniash, Wiley Interdiscip. Rev.: Nanomed. Nanobiotechnol., 2011, 3, 47.

35 F. O. Fallasotelo, M. A. Rizzuto, M. H. Tabacnicks, N. Added and M. D. L. Barbosa, Br. J. Appl. Phys., 2005, 35, 761.

36 F. Lippert and R. J. Lynch, Arch. Oral Biol., 2014, 59, 704.

37 J. M. TenCate, K. A. Dundon, P. G. Vernon, F. A. Damato, E. Huntington, R. A. Exterkate, J. S. Wefel, T. Jordan, K. W. Stephen and A. J. Roberts, Caries Res., 1996, 30, 400.

38 L. Li, Crit. Rev. Oral Biol. Med., 2003, 14, 100.

39 M. Kakei, T. Sakae, M. Yoshikawa and N. Tamura, Ann. Anat., 2007, 189, 175.

40 P. Güneri, E. Alpöz, J. B. Epstein, H. Çankaya and M. Ateș, Special Care in Dentistry, 2011, 31, 123.

41 R. K. Gudipaneni, R. V. Kumar, J. Govada, S. Peddengatagari and Y. Duddu, J. Clin. Diagn. Res., 2014, 8, 18. 